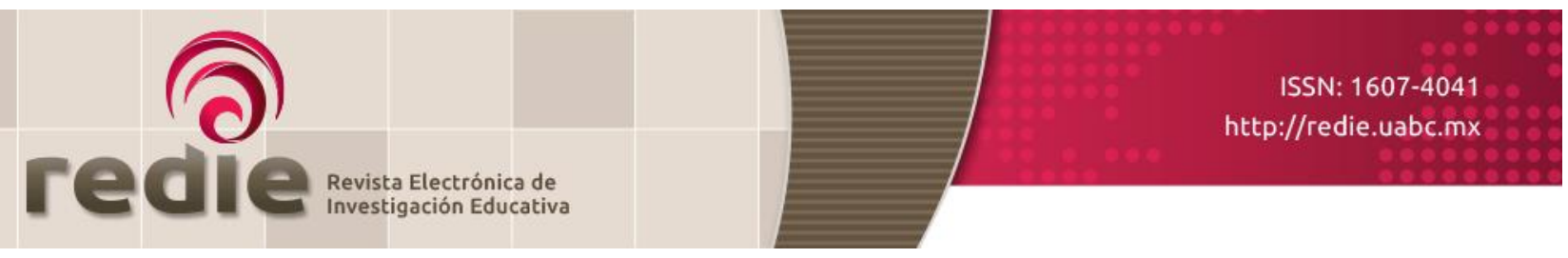

Vol. 20, Núm. 1, 2018

\title{
El cambio climático en las representaciones sociales de los estudiantes universitarios
}

\section{Climate Change in University Students' Social Representations}

\author{
Raúl Calixto Flores (*) rcalixto@upn.mx \\ (*) Universidad Pedagógica Nacional \\ (Recibido: 10 de junio de 2016; Aceptado para su publicación: 10 de noviembre de 2016) \\ Cómo citar: Calixto, R. (2018). El cambio climático en las representaciones sociales de los estudiantes universitarios. Revista \\ Electrónica de Investigación Educativa, 20(1), 122-132. https://doi.org/10.24320/redie.2018.20.1.1443
}

\begin{abstract}
Resumen
El objetivo de este artículo es describir los resultados de la investigación sobre las representaciones sociales del cambio climático en una muestra de 105 de estudiantes de la Universidad Pedagógica Nacional (México). Se trabajó por medio de cartas asociativas, fundamentadas en la asociación libre de palabras a partir de un término inductor, un cuestionario con preguntas abiertas y cerradas y entrevistas cualitativas a partir de la presentación de imágenes relacionadas con el cambio climático. Los resultados muestran que los universitarios poseen representaciones sociales del cambio climático que comprenden contenidos hegemónicos (referidos principalmente a los efectos y a las alternativas educativas para enfrentarlo), emancipados (que incorporan cuestiones relacionadas con las causas), y polémicos (presentan aspectos emocionales relacionados con el fenómeno).
\end{abstract}

Palabras clave: Educación ambiental, representación social, universitarios.

\section{Abstract}

The objective of this paper is to describe the results of a study on social representations of climate change in a sample of 105 students from the National Pedagogical University (Mexico). Work was carried out using associative cards, with free association of words from a stimulus word, a questionnaire with open-ended and closed-ended questions, and qualitative interviews that drew on climate change-related images shown to subjects. The results show that university students hold social representations of climate change that include content that is hegemonic (mainly relating to the effects of climate change and ways to address it in education), emancipated (integrating issues relating to causes), and polemical (they feature emotional aspects associated with the phenomenon).

Keywords: Environmental education, social representation, university students. 


\section{Introducción}

Las actividades humanas intensivas que atentan contra los ciclos naturales del planeta constituyen la principal causa del origen del Cambio Climático (cc). Alteraciones que se manifiestan de múltiples formas y que impactan negativamente la calidad de vida de millones de personas; ante esta situación, resulta prioritario realizar investigaciones orientadas a un mayor y mejor conocimiento de cómo los sujetos describen y comprenden las causas y efectos del cc. La investigación que se describe en este artículo buscó la identificación y caracterización de los contenidos de las representaciones sociales (RS) del cambio climático (cc) en una muestra de estudiantes de las licenciaturas escolarizadas de la Universidad Pedagógica Nacional (UPN).

Los efectos del cc se multiplican; a diferencia de hace algunas décadas la humanidad se acerca al punto de no retorno, es decir, las alteraciones ambientales están ocasionando daños irreversibles que trastocan los ciclos fisicoquímicos y atmosféricos, lo que a su vez altera el clima y pone en riesgo la supervivencia de miles de formas de vida.

Al respecto, Lovelock (2007) menciona que el planeta ya se encuentra en una situación tal, que los esfuerzos que se realicen por reducir los efectos del Cc no tendrán éxito. Por otra parte, Randers (2012), sostiene que en la segunda mitad del siglo XXI el planeta tendrá una crisis ambiental severa y las vías sustentables ya no estarán al alcance de la humanidad.

Las condiciones del medio ambiente no sólo se están alterando, sino que se están perturbando a un ritmo acelerado, con transformaciones diversas ocurriendo a la vez, las cuales no permiten adaptaciones progresivas ni de los procesos naturales, ni de los seres humanos; cada vez más se están alterando las condiciones esenciales para la vida y el cambio climático provoca también todo tipo de modificaciones como las biológicas y las geográficas en el planeta. (De Ambrosio, 2014, p. 55).

El problema del cc es de tal magnitud que se requiere de la intervención no sólo de las Ciencias Ambientales; en el ámbito educativo -y específicamente en el campo de la educación ambiental- se ha desarrollado la línea de investigación de las RS del CC.

Las RS centran su atención en el conocimiento de sentido común, referido a grandes parcelas de la realidad, como el mundo físico, el mundo de la vida o el mundo social, o aspectos muy limitados -como una práctica profesional específica.

Desde la Psicología Social, las representaciones adquieren el atributo de sociales:

Lo social de las representaciones es un atributo relacional que se gesta en las interacciones entre personas, grupos y objetos, pudiendo estos últimos ser materiales, imaginarios o simbólicos. Finalmente, las representaciones comunes surgen en los procesos de interacción social que al nombrar los objetos y atribuirles valor, están siendo co-construidos. (Wagner, 1995, s/p).

Las RS consideran la génesis del conocimiento social dentro de un marco cultural y de interacción que los medios de comunicación contribuyen a formar.

Las representaciones sociales son una red de interactuar conceptos e imágenes cuyo contenido evoluciona continuamente con el tiempo y el espacio. Cómo la red evoluciona depende de la complejidad y la velocidad de comunicación, así como en los medios de comunicación disponibles. Y sus características sociales están determinadas por las interacciones entre los individuos y/o grupos, y el efecto que tienen sobre sí como una función de la relación que les une. (Moscovici, 1988, p. 220).

Las RS son consideradas en esta investigación como una forma de conocimiento de sentido común y práctico, que permite obtener una mirada funcional del mundo; comprenden una serie de nociones que 
permiten identificar la visión que tiene el sujeto acerca de cierto objeto. Estas representaciones le dan el sentido a la realidad y son un puente con la práctica; las RS comprenden algo que se presenta y algo que está en lugar de otra cosa, por lo que juegan un papel importante en la comunicación, fungen como vínculo entre el representante y el representado y se encuentran en el lenguaje cotidiano de los sujetos.

El uso de las RS como herramienta de análisis en la investigación social aplicada permite develar constructos cognitivos sobre el medio ambiente y, en parte, explican las conductas asociadas a dichos constructos (D’ Amato, 2012, p. 46).

Las RS del Cc han sido identificadas en diversos sectores de la población, como han documentado BBC World, 2007; Jovchelovitch, 2001; Leiserowitz, 2007; Leiserowitz, Maibach y Roser-Renouf, 2010; Meira, 2002, 2009, 2012; Meira у Arto, 2008; Meira y Arto, 2013; Meira, Arto, Heras y Montero, 2011; Urbina, 2006; entre otros.

La BBC (2007), al encuestar a 22,000 personas en 21 países, reveló que predomina un porcentaje alto que considera a la actividad humana como una causa significativa del cc: $77 \%$ en Canadá; $71 \%$ en Estados Unidos; 94\% en México; 88\% en Brasil; 85\% en Chile; 93\% en España; 92\% en Italia; 89\% en Francia; 87\% en Alemania; $79 \%$ en Rusia; $78 \%$ en Gran Bretaña; $70 \%$ en Turquía; $66 \%$ en Egipto; $72 \%$ en Nigeria; $72 \%$ en Kenia; $91 \%$ en Corea del Sur; $87 \%$ en China; $81 \%$ en Australia; $76 \%$ en Filipinas; $71 \%$ en Indonesia; $47 \%$ en India. Lo diferentes porcentajes se explicaan por las diferentes posibilidades de acceso a la información.

Estos resultados muestran que el $\mathrm{cc}$ es un tema de interés, conocido por un alto porcentaje de la población, en el caso de México, el porcentaje de personas que asocian las actividades humanas con el cc, es uno de los más altos.

En México, Urbina (2006) encontró que la mayor parte de las personas (46\%) considera conocer "algo" sobre el cc; $36 \%$ "muy poco" y 10\% "mucho", y 7\% dice conocer "mucho". En este caso, el autor atribuye como factor de estas diferencias la escolaridad de los sujetos, ya que determina las posibilidades diferenciadas en el acceso a la información relacionada con el cc.

Estos resultados nos llevan a considerar a los estudiantes universitarios como una población que puede acceder con mayor facilidad a la información sobe el cc, ya que se encuentra mejor informada que la "gente común".

La investigación de las RS del CC en las instituciones de educación superior proporciona elementos para comprender si la educación ambiental ha promovido en los estudiantes la generación de comportamientos favorables al medio ambiente. Las RS del cc en los estudiantes de educación superior han sido investigadas por Boyes y Stanisstreet, 1992; Cabecinhas, Lázaro y Carvalho, 2008; Correa, 2012; Dove, 1996; Gautier, Deutsch y Rebich, 2006; González-Gaudiano y Maldonado, 2012; Jaspal, Nerlich y Cinnirella, 2014; Meira, 2012; Meira y Arto, 2013; Padilla, 2010; Rebich y Gautier, 2005; Rebich, Deustc y Gautier, 2006, y Terrón y Bahena, 2015, entre otros. En la tabla I se muestran algunos de los principales resultados de las investigaciones referidas. 
Tabla I. Investigaciones previas referidas a las Rs del cc en la población universitaria

\begin{tabular}{|c|c|}
\hline Investigaciones & Resultados de las RS de los estudiantes \\
\hline $\begin{array}{l}\text { Dove, 1996; Meira 2012; Terrón y Bahena, 2015; } \\
\text { Rebich y Gautier, } 2005 .\end{array}$ & $\begin{array}{l}\text { Desconocen o tienen poca claridad sobre la problemática } \\
\text { del cc. }\end{array}$ \\
\hline Dove, 1996; Меiга, 2012. & $\begin{array}{l}\text { Encuentran relaciones entre el efecto invernadero con el } \\
\text { agotamiento de la capa de ozono. }\end{array}$ \\
\hline Rebich y Gautier, 2005. & $\begin{array}{l}\text { Consideran el cc como resultado del agujero de la capa de } \\
\text { ozono. }\end{array}$ \\
\hline $\begin{array}{l}\text { Boyes y Stanisstreet, 1992; Dove, 1996; } \\
\text { Meira, 2012; Meira y Arto, 2013; } \\
\text { Rebich y Gautier, 2005; Terrón y Bahena, } 2015 .\end{array}$ & $\begin{array}{l}\text { Identifican una fuerte asociación entre la existencia del } \\
\text { agujero de la capa de ozono y el cc. }\end{array}$ \\
\hline Rebich y Gautier, 2005; Ramírez, 2014. & $\begin{array}{l}\text { Predomina la identificación del efecto invernadero } \\
\text { originado por las actividades humanas, pero no como } \\
\text { un fenómeno natural. }\end{array}$ \\
\hline Meira y Arto, 2013. & Desconocen o minimizan las causas naturales del cc. \\
\hline Correa, 2012; Gautier, Deutsch y Rebich , 2006. & $\begin{array}{l}\text { Desconocen las causas que originan los gases efecto } \\
\text { invernadero. }\end{array}$ \\
\hline $\begin{array}{l}\text { Boyes y Stanisstreet,1992; Correa, 2012; } \\
\text { Meira y Arto, 2013; Padilla, 2010; Ramírez, } 2014 .\end{array}$ & $\begin{array}{l}\text { Encuentran confusiones entre las causas y efectos } \\
\text { del cc. }\end{array}$ \\
\hline $\begin{array}{l}\text { González-Gaudiano y Maldonado, 2012; Jaspal, } \\
\text { Nerlich y Cinnirella, 2014; Montoya y Acevedo, } \\
\text { 2013; Moscardo, 2013; Ramírez, } 2014 .\end{array}$ & $\begin{array}{l}\text { Identifican barreras y disposiciones para actuar ante } \\
\text { el cc. }\end{array}$ \\
\hline Cabecinhas, Lázaro y Carvalho, 2008. & $\begin{array}{l}\text { Describen la existencia un alto nivel de preocupación y por } \\
\text { los riesgos hacia los posibles efectos del cc. }\end{array}$ \\
\hline
\end{tabular}

Fuente : Elaboración propia a partir de los resultados de las investigaciones referidas.

En este conjunto de investigaciones se observan diferentes aspectos de las RS del cc en los estudiantes universitarios; es decir, el cc ha pasado a formar parte del conocimiento de sentido común en este sector de la población; con estas representaciones, los estudiantes dialogan, explican y llegan a tomar decisiones en su vida cotidiana respecto al cc.

\section{Metodología}

La investigación corresponde a un estudio exploratorio y descriptivo en el que se privilegia el enfoque procesual de las RS (Banchs, 2000) desarrollado por Jodelet (2000) en estrecha cercanía con la propuesta original de Moscovici (1979); se manejan datos cualitativos, pues interesa descubrir los significados no manifiestos de la experiencia de los estudiantes. La investigación se enmarca en el campo de la educación ambiental con los objetivos de: 1) Identificar los contenidos de las RS del cc y, 2) caracterizar los contenidos presentes en las RS del cc.

La perspectiva metodológica privilegió el empleo de técnicas cualitativas (Martínez, 2004) por medio de diversos instrumentos: cartas asociativas, fundamentadas en la asociación libre de palabras a partir de un término inductor, los sujetos expresan las palabras libremente (Abric,1994); un cuestionario con preguntas abiertas y cerradas (Meira, 2003); y entrevistas cualitativas a partir de la presentación de imágenes relacionadas con el cc (Abric, 1994; Dowdall y Golden , 1989).

La carta asociativa abarcó cuatro niveles de asociación a partir del término inductor "cambio climático"; el cuestionario consistió en diez preguntas, ocho cerradas y dos abiertas referidas a los aspectos generales del cc: origen, causas y consecuencias; y el guión de entrevista incluyó cuatro preguntas generadoras, fundamentadas en la presentación de imágenes referidas al cc (las imágenes fueron seleccionadas previamente por 20 estudiantes de la licenciatura en Pedagogía de la UPN y 20 estudiantes de la licenciatura en Arquitectura de la UNAM). 
Los instrumentos empleados se pilotearon previamente con una muestra de 20 estudiantes de la licenciatura en Pedagogía de la UNAM. La aplicación definitiva se realizó de forma escalonada, primero la carta asociativa, en un segundo momento el cuestionario y por último la entrevista. Los instrumentos empleados facilitaron el diálogo y dieron la posibilidad de que surgieran nuevas interrogantes; las categorías emergieron de la organización y sistematización de la información.

La investigación se realizó en la UPN, unidad Ajusco, localizada en el sur de la Ciudad de México, donde se ofrecen cinco licenciaturas escolarizadas: Administración Educativa (Ae), Educación Indígena (Ei), Pedagogía (Pg) y Psicología Educativa (Pe). La muestra fue selectiva hasta completar 21 estudiantes por licenciatura, con base en tres características: estar inscritos en el quinto o séptimo semestre, manifestar interés en participar en el estudio y tener disponibilidad de tiempo. Los resultados obtenidos no son generalizables, pero dan pauta para identificar las características de las RS del cc en la población universitaria. De Ae participaron 10 mujeres y 11 hombres; de la licenciatura en Ei 14 mujeres y 7 hombres; de Pg 18 mujeres y 3 hombres; de Pe 7 mujeres y 14 hombres, y de Se 10 mujeres y 11 hombres.

\section{Resultados}

En la presentación de los resultados se utilizan las siglas de cada licenciatura, anotando los porcentajes de las respuestas; se transcriben textualmente algunas de éstas, identificando al estudiante con un número. Los resultados se presentan considerando los contenidos propuestos por Moscovici (1988) y retomados por Rodríguez y García (2007): hegemónicos, emancipados y polémicos.

- Contenidos hegemónicos son los más compartidos, se hacen visibles por medio de enunciados afirmativos y descriptivos, se asumen como naturales; son en lo que existe un mayor consenso.

- Contenidos emancipados se refieren a creencias y valores que sostienen subgrupos, que se comparten en un momento dado, y de los cuales no hay consenso en el grupo en general.

- Contenidos polémicos son aquellos que emergen y que pueden formar parte de los otros tipos de contenidos, en términos de reconocimiento, aceptación y legitimidad.

Los contenidos referidos se identifican en la representaciones de los estudiantes; es necesario considerar que la presencia de los contenidos emancipados y polémicos pueden cambiar de forma paulatina de acuerdo a la información que los estudiantes asimilan, principalmente por la influencia de los distintos medios de comunicación y los procesos de escolarización; en tanto que los contenidos hegemónicos se encuentran más arraigados en el núcleo figurativo de las representaciones, y son más difíciles de cambiar. Pero también se observa la ausencia de una serie de contenidos relevantes para comprender la problemática del cc que aún no son incorporados en el lenguaje cotidiano de los estudiantes. La caracterización de los contenidos permite identificar el sentido y significado que los estudiantes le imprimen al cc.

A diferencia del enfoque estructural, en esta investigación no se utilizó la carta asociativa para definir los procesos cognitivos y estructurales, sino para identificar la conformación básica del núcleo figurativo de las representaciones.

En la elaboración de las cartas asociativas los estudiantes de cada licenciatura utilizaron un número variable de palabras distintas: Ae 234 palabras; Ei 255 palabras; Pg 222 palabras; Pe 299 palabras y Se 220 palabras. El número de palabras utilizadas, además de indicar que los estudiantes cuentan con información sobre el cc, permite analizar las distintas asociaciones realizadas e ir identificando las palabras que corresponden a determinados tipos de contenidos.

Las cinco palabras más utilizadas por los estudiantes de las cinco licenciaturas al elaborar las cartas asociativas fueron: contaminación (41.90\%), calor (32.38\%), agua (30.47\%), lluvia (27.61\%) y frío (27.61\%); pero de acuerdo a la licenciatura, el orden fue diferente y se incluyeron otras palabras. 
Tabla II. Palabras más utilizadas

\begin{tabular}{|c|c|c|c|c|}
\hline $\begin{array}{l}\text { Administración } \\
\text { de la educación }\end{array}$ & Educación indígena & Pedagogía & Psicología educativa & $\begin{array}{c}\text { Sociología } \\
\text { de la educación }\end{array}$ \\
\hline Contaminación & Calor (61.90\%) & Contaminación & Lluvia (66.66\%) & Calor (57.14\%) \\
\hline$(52.38 \%)$ & Agua (52.38\%) & $(90.47 \%)$ & Inundaciones (57.14\%) & Basura (38.09\%) \\
\hline Muerte (47.61\%) & Frío (52.38\%) & Agua (71.42\%) & Frío $(47.61 \%)$ & Frío (38.09\%) \\
\hline Basura (33.33\%) & Lluvia (42.85\%) & Calor (61.90\%) & Enfermedades (42.85\%) & Muerte (38.09\%) \\
\hline Agua (28.57\%) & Contaminación & Muerte (42.85\%) & Calor $(42.85 \%)$ & $\begin{array}{l}\text { Contaminacion } \\
(2857 \%)\end{array}$ \\
\hline Lluvia (28.57\%) & $(38.09 \%)$ & Personas(42.85\%) & & \\
\hline
\end{tabular}

Fuente : Elaboración propia con información de las cartas asociativas.

En los resultados de las cartas asociativas se observó una clara relación entre la contaminación y el cambio climático; emergieron también palabras con un componente emocional, como tristeza y miedo. Al analizar las asociaciones válidas de las palabras utilizadas por los estudiantes de cada licenciatura es posible identificar la conformación de las RS del CC; asociaciones como contaminación-calor-basura; agualluvia-inundaciones; enfermedades-muerte-personas, son de las más frecuentes. Estas asociaciones forman parte de los contenidos hegemónicos, que son los compartidos por la mayoría de los estudiantes de las cinco licenciaturas.

Las asociaciones como lluvia-agua-animales en Ae; calor-plantas-clima en Ei; lluvia-personas-agua en Pg; calor-peligro-animales en Pe, o frío-plantas-destrucción en Se, contienen contenidos emancipados que corresponden a los compartidos en los subgrupos y de los cuales no hay consenso en el grupo en general.

Y las asociaciones muerte-sequía-tristeza; temperatura-clima-miedo; extinción-desinterés-tristeza; impotencia-tragedia-vida, y cambio-inconciencia-corrupción -que son menos fecuentes, pero están presentes en las cinco licenciaturas- corresponden a los contenidos polémicos, que se caracterizan por ser contenidos emergentes y controversiales.

En las distintas cartas asociativas realizadas por los estudiantes se observa el predominio de los contenidos hegemónicos referidos a los efectos del cc; en los contenidos emancipados, que existen en una menor proporción, prevalecen las confusiones en los vínculos entre las causas y efectos del cc, y en los contenidos polémicos, que son menos todavía, se observa la incorporación de aspectos emocionales. Para ejemplificar, cada uno de los contenidos transcriben de forma textual algunas de las respuestas de los estudiantes, derivadas de la entrevista cualitativa, a partir de la observación de imágenes relacionadas con el cc.

\subsection{Contenidos hegemónicos}

Me siento incómoda por no poder hacer nada ante lo que está pasando, ocurre un desequilibrio ambiental, en algunos lugares hay exceso de calor y produce sequías, y en otros lugares las intensas lluvias que provocan inundaciones, lo peor es que los únicos afectados son las personas que habitan estos lugares. (Se/13).

Las asociaciones más comunes que emplean los estudiantes observan los efectos del cc. En los estudiantes de las cinco licenciaturas se identifican contenidos de los efectos del cc, tanto para el medio ambiente natural como para las sociedades humanas. Más del $90 \%$ reconoce que el cc contribuye al incremento de los problemas en el suministro de agua potable, los huracanes y tornados, la pérdida de la biodiversidad, las sequías, el nivel del mar, la desertificación, la reducción del potencial agrícola y la incidencia de los incendios forestales. Contenidos hegemónicos que coinciden con las representaciones identificadas en los estudiantes de otras universidades (Boyes y Stanisstreet, 1992; Dove, 1996; Meira, 2012; Rebich y Gautier, 2005; Terrón y Bahena, 2015). 


\subsection{Contenidos emancipados}

También se van incorporando elementos que asocian las causas con los efectos del cc.

Cada vez nos quedamos más pobres, sin economía, sin la naturaleza, como un reflejo de lo que estamos perdiendo... todo se acaba, el agua se acaba, los animales se extinguen... no tenemos futuro. (Pe/17).

En las respuestas de los estudiantes se observa la incorporación de aspectos más globales, se establecen algunas relaciones entre las causas y efectos del cc.

El origen del cc obedece a procesos naturales y principalmente a las acciones humanas de distintas formas, sin embargo, aún algunos estudiantes atribuyen el origen sólo a causas naturales, confusión que persiste en los estudiantes de la UPN.

En los contenidos emancipados de los estudiantes de licenciatura se observan confusiones en cuanto al origen del CC, $42.85 \%$ no identifica la causas del CC; la mayoría de los estudiantes de las licenciaturas en Administración en Educación, Pedagogía y Sociología de la Educación consideran que el cc es provocado sólo por las actividades humanas (71.42\%). En la licenciatura de Educación Indígena se observan posiciones diferentes, para el $42.85 \%$ de los estudiantes el origen del cc es por causas naturales y el $38.09 \%$ lo atribuye a las actividades humanas.

Pocos estudiantes reconocen como causas del origen del cc los gases emitidos por las erupciones volcánicas, los incendios de grandes extensiones de selvas y bosques, los procesos de respiración y descomposición de animales y plantas en la Tierra y en los océanos; un mayor número de estudiantes reconoce como causas del cc el uso desmedido de los combustibles fósiles, la tala desmedida, la contaminación de mantos acuíferos, el agotamiento de los suelos fértiles y, en menor medida, el aumento en el consumo de la carne bovina. Datos similares son reportados por Boyes y Stanisstreet (1992) у Meira y Arto (2013).

\subsection{Contenidos polémicos}

Algunas de las expresiones de los estudiantes se asocian a emociones que son inherentes al cc y, gradualmente, terminan formando parte de las representaciones:

Al saber sobre el cambio climático me causa tristeza, angustia, temor, una sensación de que en este camino nos vamos a destruir, no hay salida. (Pg/9).

Las RS se vinculan con distintas emociones generadas al observar comportamientos poco favorables al medio ambiente; las expresiones emocionales pueden formar parte de los otros tipos de contenidos:

Debido a la corrupción hay sobrexplotación de los recursos naturales, enfrentamos consecuencias como la reforestación, sequías extremas, explotación de los mantos acuíferos, contaminación de los ríos debido a la industria y al mal uso de los recursos naturales. (Ae/3).

Los estudiantes manifiestan una preocupación por las condiciones actuales y futuras del planeta, pero también coinciden en que predomina una falta de credibilidad en las acciones que se realizan al respecto.

Las emociones constituyen contenidos fundamentales de las representaciones, no se reducen a reacciones o respuestas instintivas, son componentes complejos que comprenden aspectos psicológicos y fisiológicos, observables en comportamientos valorativos. Para Gutiérrez (2013), "las emociones son inseparables de toda producción subjetiva humana, en este sentido son constituyentes de las propias representaciones sociales". (p. 28). 
Vistas desde esta perspectiva, las emociones forman parte de los contenidos polémicos de las RS, ya que son difíciles de aprehender y tienen una relación estrecha con las actitudes que poseen los estudiantes:

[lo] que está pasando en nuestro planeta sólo es consecuencia de lo que hemos provocado por nuestra inconciencia, ¿qué pasará en el futuro?, lo mismo. (Pe/20).

La toma de conciencia por medio de la educación es una de las vías que los estudiantes reconocen como relevantes para incidir sobre el cc.

\subsection{Ausencia de contenidos}

Una alta proporción de estudiantes desconoce aspectos importantes del cc: el 79\% no cuenta con información relacionada con los "sumideros de carbono" y el $80.96 \%$ sobre el "comercio de emisiones de carbono"; es desconocido para un gran porcentaje que el fomento de los sumideros de carbono y el comercio de emisiones de carbono son estrategias instrumentadas para propiciar la sustentabilidad del planeta, y con ello mitigar los efectos del cc.

El 46.67\% de los estudiantes presenta dudas respecto a los gases efecto invernadero, sólo 14.28\% puede identificar al dióxido de carbono $\left(\mathrm{CO}_{2}\right)$, metano $\left(\mathrm{CH}_{4}\right)$, óxido nitroso $\left(\mathrm{N}_{2} \mathrm{O}\right)$, Ozono $\left(\mathrm{O}_{3}\right)$, Clorofluorocarbonos, (CFC), Hidroclorofluorocarbonos (HCFC) e Hidrofluorocarbonos (HFC), como parte de estos gases; y 34.29 \% de los estudiantes no logra explicar cómo ocurre el efecto invernadero.

Respecto al Protocolo de Kioto, 41.90\% indicó cuenta con alguna información; sobre el panel intergubernamental del cambio climático, 34.28\% identificó las funciones de este organismo; $42.85 \%$ de los estudiantes reconoce la importancia de la "Carta de la Tierra"; 20.95\% identifica las acciones que realiza el "Centro Mario Molina" y 11.42\% reconoce a la "huella ecológica" como una estrategia para identificar el impacto ambiental de las actividades humanas, pero ninguno mencionó la "huella hídrica" o la "huella de carbono".

Otros aspectos que los estudiantes conocen poco son los referidos a los acuerdos internacionales, estrategias y programas -a nivel internacional y nacional- para mitigar los efectos del CC, sólo 18.09\% de los estudiantes sabe algo sobre la Convención Marco de las Naciones Unidas sobre el Cambio Climático.

La RS del CC no se puede delimitar físicamente, pero sí reconocer sus causas, efectos y alternativas de solución; los efectos constituyen en las RS de los estudiantes de la UPN los principales contenidos hegemónicos. Y al igual que Jaspal, Nerlich y Cinnirella (2014) reconocen el predominio de componentes antropogenéticos, en este sentido los medios de comunicación y las instituciones de educación superior pueden generar acciones para hacer visible un problema de carácter global a la población en general, que Meira (2009) describe como "deslocalizado en el tiempo y en el espacio y demasiado abstracto". En la investigación con los estudiantes de la UPN se identifica una mayor proporción de contenidos sobre los efectos del cc que de las causas, coincidiendo con los resultados obtenidos por González-Gaudiano y Maldonado (2012).

Los estudiantes no tienen conocimiento sobre cuestiones como los gases de efecto invernadero -entre otros contenidos que para la mayoría de los estudiantes resultan confusos-; los resultados coinciden con los hallazgos de Reusswig y Meyer-Ohlendorf (2014) y Terrón y Bahena (2015). Los estudiantes de la UPN, muestran desconocimiento sobre aspectos referidos a la política y gestión ambiental, y de leyes y programas del cc en México; desconocen también los aspectos referidos al "comercio de emisiones de carbono" y los "sumideros de carbono", resultados similares a los reportados por Meira (2013), y un aspecto más de coincidencia con los trabajos de Meira y Arto (2013) es el referido a las emociones y valoraciones de carácter negativo e imágenes pesimistas. La identificación de las emociones como contenidos polémicos de las RS emergen como un aspecto relevante para comprender el sentido que le imprimen los estudiantes al cc. 
Por otra parte, los resultados expuestos coinciden en varios aspectos con los reportes de otras investigaciones de las RS del Cc en la población universitaria, pero también aportan nuevos elementos para comprender la dinámica en los contenidos de las RS, y la importancia de tomar en cuenta los factores emocionales como parte de los contenidos polémicos.

\section{Discusión y conclusiones}

Entre las principales características de los contenidos identificados en las RS del cc se encuentran la conjunción, la complementariedad, las emociones y la capacidad de transformación.

Conjunción porque se encuentran enlazados contenidos de distintas disciplinas, no sólo de las Ciencias Sociales y Humanas, sino también de las Ciencias Naturales, aunque el manejo de estos últimos es escaso y en ocasiones se identifican confusiones; complementariedad porque en las representaciones se combinan los contenidos hegemónicos, emancipados y polémicos, aunque predominan los primeros; las emociones se encuentran presentes como contenidos polémicos, pero en su gran mayoría en un sentido de "fatalidad" o "pesimismo", que suele llevar a la inactividad o desinterés. Y la característica de la capacidad de transformación constituye un proceso inherente a la dinámica de las RS. De acuerdo con Pozo (2007) es posible la reestructuración de las representaciones; en este sentido, que a partir de la identificación de los contenidos que forman el núcleo figurativo de una representación se pueden generar experiencias orientadas a reaprender, y adoptar nuevas prácticas, que en un mediano o largo plazo pueden formar parte de los contenidos hegemónicos del núcleo figurativo.

En las RS identificadas se observa el predominio de contenidos relacionados con los efectos; estas representaciones pueden enriquecerse o transformarse por medio de diversas tareas de la educación ambiental, como la investigación, formación, actualización, difusión y divulgación. La educación ambiental para el cc es importante en la educación básica, también lo es en la educación media superior y superior, así como en los distintos ámbitos de la educación y comunicación.

\section{Referencias}

Abric, C. (Coord.) (1994). Prácticas sociales y representaciones. México: Ediciones Coyoacán.

BBC World (25 de septiembre de 2007). All countries need to take major steps on climate change suggests global poll. Recuperado de

http://www.bbc.co.uk/pressoffice/pressreleases/stories/2007/09 september/25/climate.shtml

Banchs, M. (2000). Aproximaciones procesuales y estructurales al estudio de las representaciones sociales. Papers on Social Representations, 9,1-15.

Boyes, E. y Stanisstreet, M. (1992). Students' perceptions of global warming. International Journal of Environmental Studies, 42(4), 287-300.

Cabecinhas, R., Lázaro, A. y Carvalho A. (2008). Communicating climate change: discourses, mediations and perceptions. Braga, Portugal: Centro de Estudos de Comunicação e Sociedade, Universidade do Minho. Recuperado de http://www.lasics.uminho.pt/ojs/index.php/climate change

Correa, I. (2012). Cambio climático y representaciones sociales entre estudiantes de educación superior. En B. Ortiz y C. Velasco (Coords.), La percepción social del cambio climático (pp. 108-122). México: Universidad Iberoamericana Puebla y Secretaría de Medio Ambiente y Recursos Naturales.

De Ambrosio, M. (2014). Todo lo que necesitas saber sobre el cambio climático. Buenos Aires: Paidós.

Dove, J. (1996). Student teacher understanding of the greenhouse effect, ozone layer depletion and acid rain. Environmental Education Research, 2(1), 89-100. 
Dowdall, G. W. y Golden, J. (1989). Photographs as data: an analysis of images from a mental hospital. Qualitative Sociology, 12(2), 183-213.

Gautier, C., Deutsch, K. y S. Rebich, S. (2006). Misconceptions about the greenhouse effect. Journal of Geoscience Education, 54(3), 386-395.

González-Gaudiano, E. y Maldonado A. L. (2012). Representaciones sociales y cambio climático, el caso de Veracruz. En B. Ortiz y C. Velasco (Coords.), La percepción social del cambio climático (pp. 82-106). México, Universidad Iberoamericana Puebla y Secretaría de Medio Ambiente y Recursos Naturales.

Gutiérrez, S. (2013). Emociones y representaciones sociales. Reflexiones teóricas. En F. Flores (Coord.). Representaciones sociales y contextos de investigación con perspectiva género (pp. 17-44). México: CRIM.

Jaspal, R., Nerlich, B. y Cinnirella, M. (2014). Human responses to climate change: social representation, identity and socio-psychological action. Environmental Communication, 8(1), 110-130.

Jodelet, D. (2000). Representaciones sociales: contribución a un saber sociocultural sin fronteras. En D. Jodelet y A. Guerrero (Coords.), Develando la cultura (pp. 8-29). México: unAM.

Jovchelovitch, S. (2001). Social representations, public life and social construction. En K. Deaux y G. Philogene (Eds.), Representations of the social: bridging theoretical traditions. Londres: Blackwell Publishing. Recuperado de http://eprints.lse.ac.uk/2649

Leiserowitz, A. (2007). International public opinion, perception, and understanding of global climate change. (Human development report 2007/2008). Nueva York: UNDP.

Leiserowitz, A., Maibach, E., Roser-Renouf, C. y Smith, N. (2010). Climate change in the American mind: Americans' global warming beliefs and attitudes. New Haven, Ст: Yale University and George Mason University.

Lovelock, J. (2007). La venganza de la Tierra. La teoría de Gaia y el futuro de la humanidad. Barcelona: Planeta.

Martínez, M. (1998). La investigación cualitativa etnográfica en educación. Manual teórico-práctico. México: Trillas.

Meira, P. A. (2012). Ideas de la gente sobre el cambio climático: una relectura. En R. Calixto (Coord.), En la búsqueda de los sentidos y significados de la educación ambiental (pp. 73-91). México: UPN.

Meira, P. A. (2009). Comunicar el cambio climático. Escenario social y líneas de acción. Madrid: Ministerio de Medio Ambiente y Medio Rural y Marino.

Meira, P. A. (2002). Problemas ambientales globales y educación ambiental: una aproximación desde las representaciones sociales del cambio climático. En M. Campillo, J. Caride y P. A. Meira (Eds.), El papel de la educación ambiental en la pedagogía social (pp. 91-133). Murcia, España: Diego Maín.

Meira, P., Arto, M., Heras, F. y Montero, P. (2011). La sociedad ante el cambio climático. Conocimientos, valoraciones y comportamientos en la población española. Madrid: Fundación Mapfre.

Meira, P. A. y Arto, M. (2013). Representaciones del cambio climático en estudiantes universitarios en España: aportes para la educación y la comunicación. Educar em Revista, 3(Especial), 15-33.

Montoya, E. y Acevedo, E. (2013). Preocupación ambiental entre población universitaria: representaciones sociales e implicaciones en temas ambientales en la Universidad de Antioquia. El Ágora, 14(1), 241-256. 
Moscovici, S. (1979). El psicoanálisis, su imagen y su público. Buenos Aires: Huemul.

Moscovici, S. (1988). Notes towards a description of social representations. European Journal of Social Psychology, 18(3), 211-250.

Padilla, I. (2010). Social representations of climate change among students from Helsinki region universities (Tesis de maestría). Universidad de Helsinki.

Pozo, J. I. (2007). Ni cambio ni conceptual: la reconstrucción del conocimiento científico como un cambio representacional. En J. I. Pozo y F. Flores (Coords.), Cambio conceptual y representacional en el aprendizaje y la enseñanza de la ciencia (pp. 73-90). Madrid: Antonio Machado libros.

Ramírez, Y. (2014). Estudio comparativo de las representaciones sociales del cambio climático en estudiantes de licenciatura para la estructuración de pautas de comunicación educativa (Tesis doctoral). Universidad Veracruzana, México.

Randers, J. (2012). 2052: a global forecast for the next forty years. EUA: Chelsea Green Publishing.

Rebich, S., y Gautier, C. (2005). Concept mapping to reveal prior knowledge and conceptual change in a mock summit course on global climate change. Journal of Geoscience Education, 53(4), 355-365.

Rebich, S., Deustch, K. y Gautier, C. (2006). Misconceptions about the greenhouse effect. Journal of Geoscience Education, 54(3), 386-395.

Reusswig F. y Meyer-Ohlendorf, L. (2014). Social representation of climate change: a case study from Hyderabad (India). Alemania: Europaeischer Hochschulverlag.

Rodríguez, T. y García, M. (2007). Representaciones sociales. Teoría e investigación. México: Universidad de Guadalajara.

Terrón E. y Bahena D. (2015). Saberes del cambio climático de los universitarios y el camino pendiente de la educación ambiental. Trabajo presentado en el XIII Congreso Nacional de Investigación Educativa. Chihuahua, México.

Urbina, S. J. y Martínez, F. J. (2006). Más allá del cambio climático. Las dimensiones psicosociales del cambio ambiental global. México: SEMARNAT/ UNAM.

Wagner, W. (1995). Description, explanation and methd in social representations research. Papers on Social Representations, 4(2), 1-21. 